Territorios 35 / Bogotá, 2016, pp. 13-35

ISSN: 0123-8418

ISSNe: 2215-7484

La fase actual del capitalismo y la urbanización en América Latina (II)

\title{
Reformas y nuevas prácticas del Estado en el desarrollo urbano después de la crisis financiera global de 2008
}

State Reforms and Practices in Urban Development after the 2008 Global Financial Crisis

Reformas e novas práticas do Estado no desenvolvimento urbano depois da crise financeira global de 2008

Peter Brand ${ }^{*}$

Recibido: 26 de febrero de 2016

Aprobado: 4 de abril de 2016

Doi: dx.doi.org/10.12804/territ35.2016.01

Para citar este artículo:

Brand, P. (2016). Reformas y nuevas prácticas del Estado en el desarrollo urbano después de la crisis financiera global de 2008. Territorios, 35, 13-35. Doi: dx.doi.org/10.12804/territ35.2016.01

* Geógrafo de la Universidad de Leeds (Inglaterra) $y$ Doctor en Diseño Urbano de la Oxford Brookes University. Escuela de Planeación Urbano-Regional, Facultad de Arquitectura, Universidad Nacional de Colombia, sede Medellín. Correo electrónico: pbrand@unal.edu.co 
Palabras clave

crisis financiera,

prácticas del Estado, politica urbana, conflicto territorial, Birmingham (Reino Unido), Medellín

(Colombia).

Keywords

Financial crisis, state practices, institutions, regulation, urban policy, territorial conflict, Birmingham (UK), Medellín (Colombia).

Palavras-chave crise financeira, práticas do Estado, politica urbana, conflito territorial, Birmingham (Reino Unido), Medellín

(Colômbia).

\section{territarias 35}

\section{RESUMEN}

Este trabajo indaga en las transformaciones de la organización y operación del Estado, y el papel de este en el desarrollo urbano, en el período posterior a la crisis financiera global de 2008 . Se argumenta que la crisis financiera reveló la falacia de la erosión del Estado nacional en la época de globalización neoliberal, en el sentido de su resurgir manifiesto como escala de regulación clave para administrar la crisis: nexo vital para el rescate del sistema financiero global y para la imposición de los ajustes consecuentes en las escalas territoriales subnacionales. Después de una revisión de tendencias en la teoría del Estado en los estudios urbano-regionales, el trabajo describe la geografía política de la crisis financiera y sus consecuencias urbanas inmediatas, para luego analizar con más detenimiento el despliegue de las políticas espaciales y los cambios institucionales que se llevaron a cabo en dos casos contrastantes: Birmingham (Reino Unido) y Medellín (Colombia). En ambos casos se proporcionan evidencias para señalar nuevos, y probablemente duraderos, procesos de recentralización de poderes de regulación, una mayor intervención directa en los asuntos locales y nuevas formas de conflicto territorial.

\section{ABSTRACT}

This paper examines recent transformations in state organization and practices following the 2008 global financial crisis, especially with regard to urban development. It is argued that the financial crisis revealed the fallacy of the erosion of the nation state under neoliberal globalization, with its manifest resurgence as the key scale of crisis administration: nexus for both the financial rescue of the banking system and the re-scaling of regulation at the sub-national scale. After a review of recent trends in state theory in urban and regional studies, the paper describes the political geography of the financial crisis before exploring in greater detail the unfolding of spatial policy and institutional change in two contrasting cases: Birmingham in the UK and Medellín in Colombia. In both cases, evidence is provided which indicates renewed and probably durable processes of recentralization of regulatory powers and greater intervention in local affairs, as well as new forms of state territorial conflict.

\section{RESUMO}

Este trabalho indaga nas transformações da organização e operação do Estado, e o papel deste no desenvolvimento urbano, no período posterior à crise financeira global de 2008. Argumenta-se que a crise financeira revelou a falácia da erosão do Estado Nacional na época de globalização neoliberal, no sentido de seu ressurgir manifesto como escala de regulação chave para administrar a crise: nexo vital para o resgate do sistema financeiro global e para a imposição dos ajustes consequentes nas escalas territoriais subnacionais. Depois de uma revisão de tendências na teoria do Estado nos estudos urbano-regionais, o trabalho descreve a geografia política da crise financeira e as suas consequências urbanas imediatas, para depois analisar com mais detalhamento a implementação de políticas espaciais e as mudanças institucionais que se levaram a cabo em dois casos constantes: Birmingham (Reino Unido) e Medellín (Colômbia). Em ambos os casos se proporcionam evidências para assinalar novos, e provavelmente duradouro, processos de recentralização de poderes de regulação, uma maior intervenção direta nos assuntos locais e novas formas de conflito territorial. 


\section{Introducción}

La crisis financiera que estalló en 2008 en los Estados Unidos y Europa condujo a la peor recesión económica desde la Gran Depresión de los años treinta, con consecuencias que todavía se sienten globalmente. Sus efectos inmediatos en el Norte fueron dramáticos en términos urbanos, con la paralización del sector de la construcción, la congelación de megaproyectos arquitectónico-urbanísticos y la agudización del déficit de vivienda, especialmente de tipo social. Además, el rescate de los bancos privados fue a costo del endeudamiento de los bancos centrales, conllevando recortes al gasto público y la implementación de políticas de austeridad que redujeron la inversión pública y gasto en servicios sociales locales.

En este trabajo se pretende indagar las implicaciones más profundas y probablemente más duraderas de la crisis financiera, en cuanto a la organización del Estado y su papel en el desarrollo urbano. La crisis financiera constituyó un shock sistémico al capitalismo globalizado y financierizado, que propulsó el Estado nacional al frente de la estrategia del manejo de la crisis, en primer lugar como locus principal de refinanciamiento de la banca privada y luego como instrumento clave de regulación de las condiciones de recuperación económi$\mathrm{ca}$, incluyendo la redefinición de la organización institucional y territorial del Estado.

Este protagonismo del Estado nacional iba en contravía a una, hasta entonces, larga tendencia de disminución de su perfil, producto de amplios procesos de privati- zación, desregulación y reordenamiento de las escalas geográficas de regulación del capital privado, tanto desde arriba (bloques económicos, tratados de libre comercio, organismos transnacionales y multilaterales) como desde abajo (regiones, ciudades y localidades) e incluso desde los lados (organizaciones privadas y no gubernamentales que cumplen funciones públicas paralelas). En medio de este proceso las ciudades, no solamente las 'globales', sino de todas las formas y tamaños, adquirieron un perfil económico, político, cultural, arquitectónico y mediático sin antecedentes en la época moderna.

El cuerpo principal del presente trabajo consiste en el análisis empírico que indaga las nuevas formas y actuaciones del Estado en relación con el desarrollo urbano, bajo el supuesto de que la crisis financiera global de 2008 impulsó una nueva etapa de organización e intervención territorial del Estado. Esta, a su vez, determinó el direccionamiento estratégico de las políticas urbano-regionales, el alcance de las prácticas de planeación espacial, la emergencia de nuevas tensiones territoriales y nuevas crisis de legitimidad gubernamental, tal como se intenta demostrar en los dos estudios de caso. Antes, sin embargo, conviene aportar algunas observaciones sobre el Estado y su abordaje en los estudios urbano-regionales.

\section{Tendencias teóricas sobre el Estado en los estudios urbano-regionales}

Aunque la pretensión del presente trabajo no va más allá de argumentar la necesidad 
de una mayor atención al Estado en el desarrollo urbano contemporáneo, es menester enunciar algunos postulados teóricos que orientan las indagaciones. Específicamente, se retoman de los debates de los años setenta, la noción general del Estado como una relación social o la "condensación material" (Poulanzas, 1980; ver también Moncayo, 1990; Pradilla, 1987) de la relación entre clases y otras fuerzas sociales. Desde esta perspectiva, más que resolver problemas sociales, el Estado tiene la función de mantener la hegemonía de ciertas clases y fuerzas sociales por medio de un aparato institucional diseñado para conducir los conflictos sociales dentro de cauces manejables. Por tanto, el Estado no es una entidad monolítica e inmutable, sino algo que moldea y está moldeado por la dinámica de estos intereses y fuerzas, dinámica que ha adquirido un nuevo impulso dentro del proceso de acumulación capitalista en el período de globalización neoliberal.

De hecho, una característica fuerte de la ampliación y consolidación del proyecto neoliberal son las reformas permanentes del Estado y la proliferación de instituciones de regulación socio-económicas a todas las escalas. Esta se debe en parte a la intensificación de la competencia en el mercado internacional que obliga a la internalización de sus efectos en el aparato del Estado nacional (Wissen \& Brand, 2011; Moncayo, 1990) de tal manera que no es tanto que el Estado ha sido vaciado de funciones, sino que se ha producido "una transformación en su 'lógica' [mediante la cual] aquellos aparatos claves para la competencia (tales como los ministerios de finanzas y los bancos centrales) ahora dominan el proceso político" (p. 6) [traducción del autor]. Si bien en los anteriores debates teóricos sobre el Estado se privilegiaban temas como los medios de consumo colectivo y el problema de la vivienda, ahora se resaltan las redes institucionales y poderes regulatorios, tal como veremos a continuación con la descripción sucinta de algunas tendencias recientes en el abordaje del Estado en los estudios urbano-regionales.

- En primer lugar, el proyecto neoliberal dirigió inicialmente su ataque contra el Estado Keynesiano mediante el impulso de la liberalización de los mercados internacionales, la privatización de las empresas públicas y la desregulación de la empresa privada. En el caso de América Latina, el Fondo Monetario Internacional fue el instrumento especialmente efectivo en imponer tales medidas mediante sus programas de ajuste estructural desarrollados en los años ochenta, con enormes consecuencias para las ciudades en cuanto una ola de privatizaciones y adquisiciones foráneas (Betancur, 2009). Si bien muchas de estas empresas fueron de carácter nacional, también las ciudades perdieron control de empresas estratégicas (en infraestructura, servicios públicos, vivienda, salud y bienestar social) para intentar ordenar espacialmente la ciudad y garantizar un mínimo de equidad social. Una buena parte de la investigación urbana en América Latina se ha 
dirigido a la privatización y sus efectos en cuanto a la reconfiguración físicoespacial, la fragmentación, la segregación y la desigualdad social.

Esta apertura de nuevos circuitos de acumulación de capital en las ciudades no era nada nuevo. Como explica Harvey (2005), las ciudades siempre han sido lugares privilegiados para la circulación de excedentes de capital financiero, desde el París de Haussmann en el siglo XIX, hasta Nueva York y Londres en los años setenta. En este último período, el auge de este 'circuito secundario' de acumulación coincidió con una masiva desindustrialización de los países del Norte, con el sector inmobiliario encargado de revivir economías locales y reconstruir imágenes urbanas hacia el sector de servicios y consumo. En este proceso el capital privado fortalecía su influencia política en la determinación de políticas urbanas, con la emergencia de la ciudad empresarial y la competitividad urbana como sus insignias principales.

- En segundo lugar la ampliación, la profundización y el creciente carácter determinante del proceso de globalización tuvieron importantes consecuencias para el gobierno territorial. Surgió el debate sobre el reescalamiento en el cual, a grandes rasgos, se argumentó que la globalización sacudió la organización escalar heredada de la época moderna, construida sobre una jerarquía de escalas ordenadas alrededor de la escala nacional. Sobre este esquema, se propuso que el proceso de globalización conlleve a la preeminencia de la escala supranacional y al resurgimiento de la escala local ('glocalización'), dejando la escala propiamente nacional en una posición secundaria como locus de poder y principio de organización de la vida económica y social. Reflexiones posteriores, sin embargo, reevaluaron el papel del Estado nacional. Inspirado en el trabajo de Lefebvre y Poulantzas, autores como Brenner (2002); Brenner, Peck \& Theodore (2010); Jessop (2000), Jessop, Brenner \& Jones (2008), llegaron a proponer que el Estado nacional había cumplido un papel crucial en la producción de la nueva espacialidad del capitalismo globalizado, entendido como una rearticulación de escalas geográficas de regulación, la redistribución de funciones y la transformación cualitativa de las funciones y prácticas en cada caso. Tal concepción relacional de la cuestión de la escala geográfica, mediante la cual las escalas geográficas se producen y transforman mutuamente, condujo a Brenner (2004) a proponer una nueva economía política de escala y la producción de "nuevos espacios estatales", en cierto sentido liberados de fronteras fijas y estables de actuación y determinados más bien por las múltiples intersecciones y dependencias entre escalas, que a su vez condicionan cualquier conjunto de iniciativas emprendidas por las ciudades mismas. Sobre estas últimas, se consolidó el argumento territarias 35 


\section{territarias 35} 18 de que las ciudades deben entenderse como "[...] campos de fuerza institucionales, posicionadas dentro y continuamente transformadas por un paisaje de reformas regulatorias de despliegue desigual y en continuo mutación, sujeto a procesos permanentes de experimentación, circulación, fracaso, (re) consideración y crisis" (Peck, Theodore \& Brenner, 2013, p. 1093) [traducción del autor].

Esta perspectiva ayuda a explicar tanto las fortalezas como las frustraciones de las organizaciones populares y de la sociedad civil. Por un lado, encuentran apoyo en el reescalamiento hacia arriba (las organizaciones no gubernamentales y las fuentes de regulación internacionales, por ejemplo en relación con los derechos humanos, la protección ambiental y, la cuestión de género); por otro lado, su campo de acción sigue enfocado principalmente en el Estado nacional y local, ahora estructuralmente inhabilitados para responder a tales reivindicaciones e incapaz de cumplir sus funciones sociales formales. En el campo de la planeación urbana, los reclamos al 'derecho a la ciudad' se desplazan hacia la judicialización de los asuntos urbanos, y la conducción de la ciudad tiende a recaer en una figura gerencial (alcalde) más que un proyecto político colectivo.

- En tercer lugar, mencionamos la corriente de análisis que replantea la cuestión de la regulación en la fase más reciente del neoliberalismo. Si bien la re-regulación emergió tempranamente como respuesta a la necesidad de establecer las nuevas y siempre cambiantes reglas del juego, autores como Braithwaite (2008), Levi-Faur (2011) y Raco (2014) argumentan que, contrario al mito de la desregulación, el nuevo orden económico consiste en una expansión geométrica de regulación, que ellos denominan 'capitalismo regulador'. Con este término se refieren a:

Una creciente delegación a la autorregulación empresarial y profesional, y a la sociedad civil, a las redes intra e internacionales de expertos, y a una creciente regulación del Estado por el Estado, en buena parte para y a través de la competencia (Braithwaite, 2008, p. 11) [traducción del autor].

Conlleva, sin embargo, la supremacía de tecnócratas y expertos (especialmente en los campos de las finanzas y el derecho, pero también en asuntos de gestión urbana), la creciente influencia del sector privado y de las grandes corporaciones, y otro paso en el distanciamiento de los gobiernos de la ciudadanía con el consecuente debilitamiento de la democracia representativa.

Las implicaciones se hacen sentir especialmente en relación con los megaproyectos de redesarrollo urbano y megaeventos como los Juegos Olímpicos y Mundiales de Fútbol, donde la suspensión de las normas democráticas locales e imposición de los intereses privados resultan en "ciudades de excepción” (Vainer, 2011), pero en 
general se manifiestan en todas las políticas urbanas que implican grandes inversiones. Construido a partir de mecanismos jurídicos y contractuales, el capitalismo regulador amplía el poder del sector privado mediante una telaraña de entidades, redes y regulaciones que restringen el campo de maniobra de los gobiernos, tanto nacionales como locales. El poder de las grandes corporaciones fue el producto y la respuesta del crecimiento regulatorio, dominado hoy por las corporaciones mismas, con más 'gobernanza' a su favor. El capitalismo regulador también subyace a la circulación internacional de políticas urbanas y las redes de ciudades, y obstaculiza cualquier proyecto urbano que diverja de la agenda neoliberal.

Sin lugar a dudas estos acercamientos teóricos alrededor de la privatización, el reescalamiento y las nuevas formas de regulación, proporcionan unas herramientas útiles para entender las características del Estado y las políticas urbanas en el nuevo milenio. Desde luego no son mutuamente exclusivas, pueden coexistir en una diversidad de combinaciones según el tiempo y el lugar, y América Latina demuestra una heterogeneidad excepcional de condiciones. Con el fin de discernir con más precisión el papel del Estado y proporcionar unos elementos de análisis que podrían conducir eventualmente a una mayor teorización al respecto, a continuación se exploran las características político-geográficas de la crisis financiera de 2008 antes de indagar sus consecuencias urbanas a través de los dos estudios de caso.

\section{La geografía política de la crisis financiera y la planeación urbana}

No es el propósito aquí entrar en detalle sobre las causas de la crisis financiera de 2007-2008, sobre las cuales existen excelentes trabajos relacionados con las prácticas predatorias de las entidades financieras, la proliferación de nuevos instrumentos financieros globales y el carácter especulativo de la industria financiera desregulada y fuera de control. Más bien centramos nuestra atención en su dimensión geográfica y sus implicaciones para la intervención estatal. Como observa Albers (2009), la crisis financiera fue un fenómeno especialmente geográfico, en el sentido de que los mercados de vivienda locales adquirieron un significado global mediante la titularización o empaquetamiento y venta de créditos hipotecarios en los mercados financieros internacionales, involucrando así países y regiones que nada tenían que ver con los orígenes directos de la crisis en los préstamos subprime en los Estados Unidos. A partir de ese momento quedó patente que el período anterior de crecimiento económico se había basado en tasas insostenibles de gastos de consumo y endeudamiento promovidas por un sistema financiero especulativo y desregulado, y la burbuja se rompió a través de los mercados de la vivienda y de propiedad raíz (Kitson, Martin \& Tyler, 2011).

La respuesta inmediata a la crisis fue un esfuerzo descomunal para rescatar los bancos. Unos pocos bancos se quebraron (siendo Lehman el más notable), pero la territarias 35 
gran mayoría fueron rescatados a un costo enorme (US\$ 1,5 trillones según el FMI, citado en Kitson, Martin, \& Tyler, 2011), hecho que produjo caos en las economías nacionales. Los gobiernos nacionales se encontraron comprometidos con niveles de endeudamiento sin precedentes y qué hacer frente a esta situación fue la cuestión que dominó los años siguientes.

El impacto inmediato golpeó fuertemente a las ciudades, pero de manera heterogénea y con consecuencias socioespaciales muy diferenciadas. En Estados Unidos, los foreclosures (extinción del derecho hipotecario) se concentraron en ciertas ciudades y sectores urbanos, con efectos especialmente duros para las familias de bajos ingresos y las minorías raciales (Aalbers, 2009; MacLeavy, 2011). En Europa, la tasa de foreclosures fue menor, pero de repente los jóvenes y las familias de bajos ingresos encontraron aún mayores obstáculos para ingresar al mercado de vivienda debido a la contracción de facilidades de crédito. Este factor también produjo una suspensión de los grandes proyectos inmobiliarios a lo largo y ancho del continente y partes del Medio Oriente, en España un millón de viviendas construidas quedaron sin posibilidad de venta, y en todo el continente millares de inversionistas, tanto individuales como institucionales, encontraron sus capitales desvalorizados o perdidos por completo.

Frente a la recesión económica, la más profunda desde los años treinta, los gobiernos y las organizaciones multilaterales se apresuraron en declarar el fin de la crisis. A finales de 2009 argumentaron que ya había pasado lo peor y que pronto se normalizaría la situación. Esto tenía algo de cierto en el caso de los Estados Unidos donde se restauró el crecimiento económico (bajo) más o menos pronto, pero otra fue la situación en Europa. Países como Grecia, España, Irlanda y Portugal fueron obligados a buscar ayuda de la Unión Europea para sortear sus deudas públicas, y el crecimiento negativo sostenido y el espectro de una recesión secundaria atormentaron el continente. La economía europea en general sigue severamente afectada, con nuevos temores de estancamiento y deflación. El efecto de todo esto sobre el comercio mundial conllevó a tasas menores de crecimiento en China, temores en cuanto a las economías emergentes y un diagnóstico reservado para la economía global.

Como observa Keil (2010), el Estado fue obligado a responder enérgicamente a la crisis financiera. Les correspondió a los gobiernos nacionales salvar la banca y enfrentar las consecuencias. Los altísimos niveles de endeudamiento público típicamente conllevaron a fuertes recortes en el gasto público, afectando especialmente al empleo en el sector público (despidos, reducción de salarios, empeoramiento de pensiones) y a las políticas sociales (de bienestar básico y servicios sociales) en una nueva ronda de "políticas de austeridad". Por otro lado, quedaron sustancialmente sin tocar el sistema financiero y su cultura de incentivos, junto con las exenciones impositivas concedidas a los más adinerados y las grandes corporaciones. La reacción pública frente a la injusticia social de todo esto condujo a 
movimientos como Occupy en Wall Street y el City de Londres, los Indignados en España y amplias protestas en Grecia y Portugal.

Desde una perspectiva urbana, una consecuencia importante fue un nuevo matiz en la estrecha relación entre las ciudades y el capital privado (Harding, Harloe \& Rees, 2010; Kirkpatrick \& Smith, 2011). La noción de gobernanza, promovida como una manera más horizontal, flexible, eficiente e incluyente para enfrentar los complejos procesos de cambio contemporáneo, se había establecido como la nueva ortodoxia de la política urbana. A través de ella el sector privado acumuló una fuerte influencia y bajo la fachada legitimadora de la gobernanza, se había desplegado una amplia gama de privatizaciones, desregulaciones y restricciones sobre la actuación estatal en cuanto a inversión pública y gasto social. El colapso temporal de este modelo de desarrollo urbano movido por el mercado inmobiliario, el empresarismo urbano y las alianzas público-privadas, resultó en una resurrección renovada casi inmediata. Las presiones fiscales fueron rebotadas a las administraciones locales y resultaron en lo que Peck (2012) llama un período de 'matoneo fiscal' y nuevos extremos de privatización, monetización y subcontratación en los Estados Unidos, citando los casos de Maywood, California y Sandy Springs, Georgia como ejemplos. En el Reino Unido, administraciones locales como Brent en Londres y el condado de Suffolk propusieron la reducción del aparato estatal local a un esqueleto administrativo (liderado por abogados y contadores) para la contrata- ción total de servicios para ser prestados por el sector privado. Si bien hace tiempo el mundo académico había advertido sobre el 'déficit democrático' del neoliberalismo, estos últimos casos de privatización radical también provocaron la resistencia ciudadana y no se han llevado a cabo todavía.

La austeridad se volvió la palabra clave en materia fiscal. En sí misma la austeridad no es nada nueva, siendo un elemento recurrente en tiempos de crisis económica (Levitas, 2012), así como un elemento común a las diferentes fases del proyecto neoliberal, caracterizado por un estado permanente de restricciones fiscales y orientación hacia el mercado (Peck, 2012). Argumenta Peck que la crisis de 2008 ha implicado un Estado más revanchista y de austeridad sistemática, conduciendo a la "incapacitación cumulativa" del Estado. Tal agenda política fue implementada con especial energía en los Estados Unidos y el Reino Unido, e impuesta a los países más reticentes a seguir la nueva ortodoxia de austeridad por las instituciones financieras multilaterales. Posteriores llamadas por parte del Fondo Monetario Internacional por ejemplo, a relajar la política de austeridad no tuvieron mayor impacto. Como observa Krugman (citado en Peck, 2012), "en el fondo la política de austeridad no tiene que ver con el endeudamiento y los déficits fiscales; se trata del empleo del pánico sobre el déficit como una disculpa para desmantelar los programas sociales". De manera similar Chang (The Guardian, 2013a) argumenta que en el caso del Reino Unido: territarios 35 
[...] el gobierno de coalición no es tan estúpido ni tan terco como parece a primera vista. Mantiene su Plan A [de austeridad] porque los recortes del gasto público no tienen que ver con el problema del déficit, sino con el desmantelamiento del Estado de Bienestar. Ninguna cantidad de evidencia económica va a cambiar su posición sobre los recortes fiscales [en línea] [traducción del autor].

Se argumenta aquí que la política de austeridad es también un asunto de la reconfiguración de la planificación espacial para facilitar, compensar y legitimar el proceso de minimización del control directo del Estado. En este sentido, vale la pena traer a colación la lectura del neoliberalismo de Harvey (2005) como 'acumulación por desposeimiento'; un proyecto de clase que involucra un ataque sostenido a la clase trabajadora y cada vez más a la clase media, la desigualdad social creciente y la concentración de la riqueza y el poder. Cada crisis permite impulsar tal proyecto político en la medida en que las políticas de austeridad se renueven contra toda lógica económica y con ceguera frente a políticas alternativas, utilizando la austeridad como motivo para reducir aún más los estándares y la calidad de vida de los pobres y profundizar la concentración de la riqueza en manos de unas élites económicas y políticas.

Lo descrito hasta ahora - tanto los sucesos reales como la teorización- hace referencia principal al mundo anglosajón y en menos grado a Europa occidental. Sin embargo, los efectos de la crisis financiera se sintieron ampliamente a causa de sus consecuencias para la economía mundial. No obstante la coherencia estructural del proyecto neoliberal, su desenvolvimiento en la práctica se reconoce como un proceso improvisado, experimental y contradictorio, hecho que se aplica también en el caso de la planeación local, campo, para los gobiernos neoliberales, de estructuras y procedimientos formales que restringen innecesariamente el desarrollo. En tiempos de crisis, la ciudad está sometida a fuerzas simultáneas de desregulación y re-regulación para facilitar la renovación de circuitos de acumulación capitalista a través del desarrollo urbano, al tiempo que la planeación urbana está utilizada para intentar reconstruir formas de estabilidad social mediante la invención de nuevos sentidos de lugar y comunidad. A continuación se examinan estos procesos en medio de una creciente intervención de los gobiernos centrales sobre la escala local.

\section{Reino Unido, Birmingham}

Igual a la mayor parte de Europa, el sistema bancario del Reino Unido fue fuertemente implicado en la crisis financiera de 2008. Los bancos principales se encontraron con la obligación de recapitalizar, fusionar o fueron efectivamente - más no jurídicamente- nacionalizados, en una operación de rescate que costó al gobierno británico una suma estimada en $£ 120$ billones (aproximadamente US $\$ 160$ billones). Se produjo una fuerte contracción del crédito y de la confianza en la economía en general, seguida por una reducción en la producción 
nacional, un aumento del desempleo y empezaron a sedimentarse los efectos a mediano plazo del endeudamiento público, asunto que dominaría la política económica en los años siguientes.

La crisis financiera - sus causas, culpables y remedios- fue el tema central de las elecciones nacionales de mayo de 2010 . El partido Conservador formó un gobierno de coalición con los Demócratas Liberales sobre una plataforma de austeridad radicalizada. La meta ostensible y obsesiva fue la de reducir la deuda pública. Hubo algunos aumentos de impuestos (IVA al 20\%, impuesto sobre las ganancias y una tasa máxima de $50 \%$ sobre los ingresos más altos), junto con la reducción real de salarios tanto en el sector privado como en el sector público (aumentos máximos del $1 \%$ anuales, por debajo de la tasa de inflación). Sin embargo, el eje principal de la estrategia consistió en una fuerte reducción en el gasto público. Esto tuvo grandes implicaciones para las ciudades, ya altamente dependientes del financiamiento central, dado que ellas son las entidades estatales responsables de una amplia gama de programas sociales. En la primera ronda de recortes fiscales, entre 2011 y 2015 los departamentos del gobierno central recibieron un recorte promedio de $11,2 \%$, mientras que el gasto de las entidades territoriales fue recortado en $26,8 \%$ (Joseph Rowntree Foundation, 2012).

En la ciudad de Birmingham, la segunda ciudad de Inglaterra y núcleo de la región metropolitana de West Midlands, todo lo anterior condujo a la pérdida inmediata de empleo en el sector público que podría llegar a alrededor de 10.000 en el período que va hasta 2017. Según un informe del municipio: "El tamaño del reto que enfrenta la ciudad cambiará el paisaje del gobierno local no solo en Birmingham, sino también en todo el país - tendremos que descontinuar numerosos servicios" (Birmingham City Council, 2012). Una encuesta de 81 municipios realizada en marzo de 2013 encontró que el presupuesto para las oficinas de planeación fue especialmente afectado, sobre todo en las grandes ciudades como Manchester (reducción de 54\%), Portsmouth (49\%) y Liverpool (35\%) (The Guardian, 2013b). Frente a tal situación, el renombrado arquitecto y exconsejero del gobierno en asuntos urbanos, Sir Richard Rogers, advirtió que se ponía en riesgo la política del 'nuevo localismo' (diseñada formalmente para entregar una mayor capacidad de decisión a las comunidades locales), dejando el sector inmobiliario en control de la agenda de desarrollo urbano (The Guardian, 2013c). Más adelante veremos cómo recortes de este tipo constituyen la última medida en una serie de reformas que tienen precisamente este propósito.

La crisis financiera fue explotada por el gobierno de coalición para también desmantelar el sistema de planeación estratégica, empezando con la planeación regional. Las Estrategias Espaciales Regionales (RSS por su sigla en inglés) fueron establecidas por el anterior gobierno laborista en los años noventa, para orientar y formar parte de los planes de desarrollo municipales. Fueron la responsabilidad de las Asambleas Regionales (constituidas por agrupaciones territarias 35

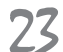


de las entidades municipales) encargadas de formular políticas holísticas de vivienda, empleo, crecimiento urbano, transporte y medio ambiente, con metas a corto, mediano y largo plazo. Paralelamente, la organización Advantage West Midlands, una de las Agencias de Desarrollo Regional bajo la orientación del gobierno central, elaboró una Estrategia Económica Regional (REE, por sus siglas en inglés). Juntas, las Asambleas Regionales y Agencias de Desarrollo Regional, constituyeron un marco claro y democrático para direccionar la inversión territorial de acuerdo con las necesidades y oportunidades de las regiones, y su agenda de renacimiento urbano produjo resultados positivos.

Todo lo anterior fue desmantelado inmediatamente por el nuevo gobierno de coalición en 2010 , en una maniobra que resonaba con la abolición de los entes metropolitanos (fortines de los gobiernos de izquierda) por parte de Margaret Thatcher en 1986. Aún en oposición el partido Conservador había propuesto "abolir por completo la escala burocrática y poco democrática de la planeación regional" (Conservative Party, 2010 [en línea]) [traducción del autor], y así procedió inmediatamente después de ganar las elecciones parlamentarias. Frente a las preocupaciones que resultaron del vacío en materia de coordinación regional, se incluyó, a última hora, una débil cláusula en el Localism Act de 2011 que estableció un 'deber de cooperar' entre las entidades de planeación involucradas en asuntos que tenían un significado más allá de lo puramente local, un mecanismo sin garantías para enfrentar territorialmente asuntos mayores como el cambio climático, la provisión de vivienda y el transporte integrado (Planning Officers Society, 2012).

La principal estrategia del gobierno central fue la de reemplazar una política regional por la acción del sector privado y 'comunidades'. Se crearon Alianzas de Emprendimiento Local (Local Enterprise Partnerships - LEPs), agencias unidimensionales (económicas) dirigidas por la empresa privada, sin ninguna responsabilidad pública directa, pero con una fuerte influencia sobre el direccionamiento de políticas de desarrollo y la inversión pública. En otro recuerdo del régimen Thatcherista, también se crearon 'zonas de emprendimiento' (Enterprise Zones) con controles mínimos de planeación.

Más que una consolidación de la planeación democrática, la abolición de las entidades de planeación regional debe ser vista como la apertura de espacios para el libre mercado. Desde la introducción de políticas neoliberales en los años ochenta, el Reino Unido se había convertido en una de las naciones más polarizadas entre los países desarrollados, con crecientes desigualdades interregionales y la concentración de la actividad económica, la inversión pública y la riqueza en Londres y el sureste de Inglaterra (Royal Town Planning Institute, 2012; Town and Country Planning Association, 2013; Office for National Statistics, 2014). Esta focalización en Londres y el descuido de las regiones fue una de las razones cruciales detrás del referéndum sobre la inde- 
pendencia de Escocia, llevado a cabo en septiembre de 2014. Asustado por las encuestas, en el período previo al referéndum los principales partidos políticos prometieron una mayor autonomía fiscal para Escocia, y una política de promoción económica en las regiones atrasadas de Inglaterra y Gales. Las disparidades regionales se volvieron, por lo menos momentáneamente, un asunto político y hasta constitucional.

En el caso de la región metropolitana de West Midlands, esta se encuentra dividida entre dos LEPs cuyos límites no corresponden a la geografía económica, sino a conveniencias políticas, lo cual dificultará aún más una respuesta coherente a los múltiples retos que enfrenta la región. Como ilustración de lo anterior, se puede citar el proyecto del ferrocarril de alta velocidad programado entre Londres y Birmingham, donde la fragilidad de planeación estratégica permite la prevalencia del interés privado en la explotación de oportunidades económicas inmediatas a costo de las necesidades más amplias de la región.

El Localism Act de 2011 también transfiere el poder decisorio sobre proyectos de infraestructura al gobierno central, apoyado en una Unidad de Grandes Proyectos de Infraestructura (Major Infrastructure and Environment Unit), una agencia ejecutiva del gobierno central, encargada de "evitar costos y demoras innecesarios en la ejecución de proyectos de infraestructura de interés nacional". Esto representa una diferenciación escalar radical y la desconexión de infraestructura de la planeación local integral (Layard, 2012).
En el caso del ferrocarril de alta velocidad, tanto las comunidades como los municipios en su área de influencia han sido desempoderados para actuar sobre proyectos de interés nacional. Una legislación posterior (Ley de Crecimiento e Infraestructura de 2013) extendió este desempoderamiento a proyectos comerciales de gran escala. En este escenario de privatización y especulación, las áreas socioeconómicas más necesitadas continuarán sufriendo de desventajas competitivas ahora institucionalizadas a través de las reformas más recientes en la gobernanza y el financiamiento local.

La estrategia de localismo representa la expresión en materia de planeación espacial de la idea nebulosa de la "gran sociedad" (big society) del partido Conservador. Forma parte de un proyecto legislativo, amplio en su alcance y complejo en su formulación, contra el "Estado grande", que pretende devolver el poder a los individuos, las comunidades y los consejos municipales (Department of Communities and Local Government, 2010). Contempla que las mismas comunidades preparen su propio plan, con el "derecho a comprar" (right to buy) los bienes locales en beneficio de la comunidad local y también a prestar servicios locales en competencia con otros actores públicos y privados. La amplia controversia suscitada entre planificadores y organizaciones sociales surge de las implicaciones prácticas detrás de la retórica. La organización nacional sindical (Trade Union Council, 2012), en un documento que recopiló la opinión de diversas organizaciones, concluyó lo siguiente: territarias 35

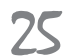


El tema común que emerge es el de una preocupación compartida sobre la agenda del gobierno en cuanto a la "gran sociedad" y los "servicios públicos abiertos", y cómo la creación de mercados de servicios públicos y un enfoque de reforma del servicio público basado en el consumidor pueda conducir a una creciente desigualdad entre y dentro de las comunidades, mercados que excluyan la participación comunitaria, la competencia a costo de la colaboración, y un localismo que descentraliza responsabilidades sin los recursos y poder correspondientes (p. l). [traducción del autor]

La tensión producida entre el control local débil y el poderoso mercado libre fue identificada como el tema crucial y la amenaza principal en un mundo posbienestar, expresado por Layard (2012) en los siguientes términos:

La Ley [Localism Act 2011] es también radical en su dependencia del capital [...] se entiende lo local como unidades y bienes. La Ley encierra vecindades para efectos de planeación, abre nuevas maneras para la prestación de servicios 'públicos', promueve la propiedad privada en vez del uso del espacio a través del derecho de compra, y facilita la construcción de vivienda nueva mediante el "derecho a construir". No toma en consideración cómo las prácticas y los procedimientos legales puedan contribuir a la comprensión y promoción de lo local por vías menos directas, ni cómo responder a los fenómenos de la exclusión y la desventaja sociales. Tampoco se preocupa por la cuestión de la justica espacial ni presta apoyo a las comunidades más pobres. En el fondo, la Ley parece continuar las prácticas del [anterior gobierno] Nuevo Laborista en el sentido de utilizar la "comunidad" para empaquetar economía y sociedad, segregando y localizando los problemas socioeconómicos generados por el neoliberalismo (p. 12). [traducción del autor].

El significado político de lo local y el localismo depende, tal como señalan Davoudi \& Madanipour (2015), del contexto en el cual se concreta. El contexto del neoliberalismo post-crisis financiera hace que "el centralismo domina al localismo en la Ley $[. .$.$] consagrado en los múltiples$ poderes, regulaciones y normas" (Institute for Local Government Studies, 2012, p. 98). La reestructuración de la planeación espacial desempodera a las entidades locales y crea un empoderamiento ficticio de la "comunidad". Visto a la luz de la crisis financiera y la respuesta nacional a ella, el resurgimiento del gobierno central en la planeación espacial local es mucho más que una medida técnica. La reestructuración y centralización del sistema de planeación contiene elementos estratégicos para reducir el gasto público, recortar el alcance de la democracia local, y posicionar el capital privado en el centro del desarrollo espacial. Si sumamos a este escenario las reducciones en el gasto público en programas sociales que empezaron a sentirse a partir de 2013, su efecto acumulativo está conduciendo a una crisis en la capacidad del Estado local

\section{territarias 35}


para incidir en las condiciones de vida de las ciudadanos.

\section{Colombia, Medellín}

En esta sección se exploran las consecuencias de la crisis financiera en un contexto muy diferente. Si en los Estados Unidos y Europa se entró en una recesión profunda y prolongada, América Latina se escapó en buena parte de sus efectos directos. El continente venía de un período de crecimiento económico sostenido desde comienzos de nuevo milenio, y apenas se produjo un parpadeo breve en 2009 para después retomar un crecimiento promedio de 3\% para la región, se redujeron los niveles de pobreza y se aumentó el gasto público (Eclac, 2012a, 2012b). El coeficiente Gini que mide la distribución social del ingreso mejoró marginalmente en la región más desigual del mundo (The Economist, 2012), en parte debida a mejoras en trabajo y salarios, pero también gracias a las transferencias condicionadas en efectivo a los más pobres (Jiménez \& López, 2012). A pesar de la persistencia de altos niveles de pobreza e informalidad en el mercado laboral y limitaciones en los servicios sociales, América Latina pudo ofrecer un trasfondo del crecimiento económico, altos niveles de inversión extranjera directa, una mejor demanda interna y más gasto público, de tal manera que las ciudades latinoamericanas se alejaron del "urbanismo de austeridad" que afligió el Norte. Sin embargo, sería erróneo concluir que la crisis financiera no tuviera consecuencias para las ciudades y las prácticas de planeación urbana.

Dentro de la diversidad latinoamericana, Colombia es considerado un país ortodoxo en materia de política económica. A lo largo de los años noventa Colombia abrió su economía, ejecutó políticas de disciplina monetaria y fiscal, e implementó paulatina y selectivamente privatizaciones y la flexibilización del mercado laboral. Se iniciaron estrategias de competitividad urbana, apoyadas por el gobierno central, pero lideradas por alianzas público-privadas a nivel urbano-regional (Brand \& Prada, 2003). Estas tendencias continuaron en el nuevo milenio, pero la crisis financiera produjo ajustes importantes a partir de 2008: más desregulación de la inversión extranjera directa, mayores incentivos impositivos para la empresa privada nacional y extranjera, y la intensificación de la búsqueda de tratados de libre comercio (con Canadá, Estados Unidos, Chile-Perú-México, Corea del Sur y la Unión Europea). También en Colombia indujeron la desindustrialización y la creciente dependencia económica en la producción primaria (petróleo, minerales, agroindustria intensiva) y el sector de servicios (formal e informal). Una mayor demanda interna alimentada por aumentos en el gasto público ha sido importante para el comportamiento económico, aunque la estrategia en general ha producido crecientes niveles de oposición por parte de grupos indígenas, ambientalistas, sindicatos laborales y movimientos populares y de izquierda.

Todo lo anterior conllevaba repercusiones importantes para las ciudades y la territarias 35 
planeación urbana. Sobre todo resaltaba las deficiencias del país en materia de infraestructura y condujo a la profundización de la privatización de infraestructuras (aeropuertos, carreteras y telecomunicaciones). También señaló el comienzo de una mayor intervención del gobierno central en los asuntos locales. Las políticas anteriores de competitividad urbano-regional lideradas por las localidades cedieron a un control más centralizado del desarrollo urbano bajo las directrices de la agenda neoliberal. Igual que en América Latina en general, la crisis financiera removió el paisaje económico al hacer más difícil el acceso a los mercados externos, y los nuevos desafíos y restricciones provocaron una respuesta más centralizada y autoritaria. En las condiciones más estrechas que siguieron al inicio de la crisis, el gobierno central se volvió más intervencionista en los asuntos urbanos, una tendencia que se ilustra a continuación.

Antes, sin embargo, conviene comentar brevemente algunas características del sistema de planeación en Colombia, cuyas bases se establecieron en los años noventa. El Estado republicano permite un alto grado de descentralización y autonomía municipal, consagrado en la Constitución de 1991 y expresado en el desarrollo del sistema de planeación en la década siguiente. El acto legislativo principal fue la Ley de Desarrollo Territorial (Ley 388 de 1997) que requiere la preparación de planes de ordenamiento territoriales. Estos contemplan un componente estratégico, un modelo de desarrollo territorial y un plan detallado de usos del suelo y normas urbanísticas, respaldadas por una gama de instrumentos jurídicos y financieros para la intervención municipal en los mercados de tierra y para la participación comunitaria y cívica. Este carácter municipalista e intervencionista, aparentemente en contravía con la tendencia de desregulación y privatización que se desplegó durante la década del noventa en áreas como la salud, las pensiones, los servicios públicos y la infraestructura, de ninguna manera afectó el boom en la industria de la construcción que se presentó en los años siguientes ni los intereses del sector privado, tal como se expone a continuación.

Como ilustración de la política neoliberal revigorizada y territorialmente intervencionista, citamos tres ejemplos de gran impacto. La primera tiene que ver con la centralización de las regalías provenientes de la explotación petrolera y minera. Antes de la crisis financiera, la mayor parte de estas quedó en manos de los municipios donde la explotación se realizaba. Como resultado, municipios pequeños en zonas alejadas del país recibieron grandes sumas de dinero para ser utilizadas en función de sus planes de desarrollo y ordenamiento territorial, y dentro de unos lineamientos generales establecidos por el gobierno central. Casos de abuso, corrupción e ineficiencia fueron utilizados como argumento para reformar radicalmente el sistema. En 2011 se introdujo la legislación para crear un fondo central, al cual cualquier municipio, productor o no de materias primas, puede acceder a estos recursos mediante la presentación de proyectos para ser evaluados por el gobierno central. De esta manera, 
el gobierno central estableció el control efectivo sobre estos fondos de desarrollo, al tiempo que introdujo el principio de competencia (entre municipios) para su aprovechamiento. En términos macroeconómicos, el control de estos fondos fue una medida importante en cuanto a la política fiscal nacional en los tiempos postcrisis.

La segunda ilustración concierne a la provisión de infraestructura, más relacionada con las ciudades medianas y grandes. El país en general sufre de un atraso grande en términos de infraestructura de transporte y comunicaciones. Esta desventaja competitiva fue resaltada por la crisis financiera y el nuevo entorno de competencia intensificada (en mercados de exportaciones existentes y nuevos), así como la negociación de tratados de libre comercio. El área de la infraestructura marcó una nueva fase de intervención directa del gobierno central en las ciudades, generalmente consultando poco a los gobiernos municipales y desconociendo las condiciones locales. Esta tendencia se había iniciado antes en el caso de los sistemas de transporte masivo. Tomando como modelo el Transmilenio (BRT o bus rapid transport) de Bogotá, que inició su funcionamiento en el 2000, el gobierno central impuso (especialmente mediante mecanismos de financiación) la replicación de este medio de transporte en siete ciudades del país, incluyendo Medellín. Mientras que la experiencia de Bogotá fue muy exitosa inicialmente (ahora sufre problemas de capacidad insuficiente y de integración operacional y tarifaria), los proyectos desarrollados en las otras ciudades sufrieron serias dificultades desde el principio en cuanto a costos, demoras y baja demanda efectiva.

Como observa Hurtado, Torres \& Mirando (2011), el mecanismo de financiación trasladó al gobierno central el control de la concepción, el diseño y el funcionamiento de los sistemas de transporte urbano. Los controles reglamentarios y la fuerza fiscal ejercidos por el gobierno central resultaron en tensiones dentro del modelo municipalista de Colombia, entre otras cosas por la poca sensibilidad a las necesidades y especificidades de cada contexto urbano. Hurtado, Torres \& Mirando (2011) concluyen:

Los sistemas integrados de transporte público en Colombia están siendo implementados con la clara intención del gobierno central de dirigir el funcionamiento del transporte urbano hacia un modelo empresarial, homogeneizado y formalizado, y con especificaciones estandarizadas a nivel técnico, estético y funcional (p. 115).

Si bien en la mayoría de los casos los sistemas BRT fueron operados por la empresa privada, Medellín fue la excepción, donde el manejo del BRT terminó en manos de la empresa Metro de Medellín, una empresa pública propiedad del municipio y operadora del metro, que en 2004 introdujo el primer sistema de cable aéreo como transporte público en los sectores pobres y escarpados de la ciudad (Brand \& Dávila, 2011; Dávila, 2013) e introdujo una línea de tranvía en 2015. Medellín es territarios 35 
única entre las ciudades colombianas en el sentido de haber desarrollado un sistema público e integrado de transporte público masivo.

El tercer caso concierne a la vivienda, donde Colombia tenía un alto déficit desde tiempos atrás, especialmente de vivienda social. A comienzos de los años noventa se creó una política con enfoque en la demanda y subsidios monetarios que resultó ser insuficiente para permitir acceso al mercado, privatizado de vivienda para la gente pobre. El gobierno argumentó que los obstáculos principales fueron la indisposición de los municipios a proporcionar suelo suficiente en los planes de ordenamiento territoriales, sus limitaciones de gestión en cuanto a la aplicación de los instrumentos jurídicos y técnicos de intervención en los mercados de suelo (Ley 388 de 1987), el desorden institucional, y el juego de intereses políticos locales, entre otras. Este tipo de racionalización fundamentó un giro en la política del gobierno central hacia la intervención directa mediante macro-proyectos de vivienda. Se abrió así el acceso de este sector de la demanda al capital privado y las grandes empresas constructoras que desde hace tiempo veían ahí un mercado amplio, pero inaccesible.

Los macroproyectos, como tales, fueron introducidos en 2007 , precisamente cuando se dio inicio a la crisis financiera global, al comienzo se enfocaron en las grandes obras de infraestructura y luego se extendieron para incluir proyectos de vivienda. Hubo una clara intención del gobierno central de fortalecer la financiación de las obras públicas por parte del capital privado, aun cuando esta implicara la imposición de los intereses macroeconómicos del gobierno central por encima de la autonomía y la democracia locales. La ley fue ampliamente criticada también por la imposición de los intereses del capital financiero y las grandes empresas inmobiliarias, el desplazamiento de comunidades y la socavación de la democracia local. La propuesta fue demandada en las altas cortes y declarada inconstitucional por razones de violación de la estructura del Estado descentralizado, la autonomía municipal y los principios de coordinación, concurrencia y subsidiaridad. Más tarde, la corte conceptuó que aquellos proyectos que habían sido aprobados e iniciados antes de esta sentencia (27 en total, con unas 218.000 viviendas) podrían continuar.

En resumen, el principal efecto de la crisis financiera en Colombia fue el de acentuar el carácter competitivo del entorno económico $\mathrm{y}$, en consecuencia, producir nuevas formas de intervención del gobierno central en los asuntos urbanos, desde el control centralizado de fondos regionales de desarrollo, hasta la competencia en la asignación de fondos a las entidades territoriales, y la intervención directa en proyectos estratégicos de desarrollo urbano. Otras medidas que pueden citarse en este sentido son la proliferación de zonas francas con mínimos controles fiscales y de planeación, así como el persistente esfuerzo de debilitar la protección ambiental mediante las llamadas "licencias exprés" para proyectos de explotación petrolera y minera. 


\section{Conclusiones}

En este trabajo se ha explorado la reconfiguración de la planeación urbana después de la crisis financiera de 2008, desde el punto de vista de las reformas en la organización y actuación del Estado. Los efectos inmediatos de la crisis sobre el desarrollo urbano en el Norte fueron bien conocidos, igual que sus consecuencias sociales, pero las implicaciones para la planeación territorial fueron menos evidentes, especialmente en el Sur. Las tendencias genéricas del Estado neoliberal identificadas al comienzo del trabajo nos alertaron a procesos como la acentuación de la privatización y la extensión del principio de competencia a esferas cada vez más amplios, el dinamismo de los arreglos multiescalares de regulación, y nuevas prácticas y loci de regulación. Igualmente, partimos de la proposición de que el rescate de la banca privada y el manejo posterior de la deuda pública iba a conducir a una mayor intervención del Estado central en los asuntos urbanos y que la crisis financiera ofreció la oportunidad de profundizar en el proyecto neoliberal de largo plazo, facilitado por la producción de la narrativa política dominante (en Europa por lo menos) que asignó la culpa por la crisis no a la banca privada, sino a los gobiernos de centro-izquierda.

$\mathrm{El}$ análisis de los dos casos contrastantes permitió precisar prácticas concretas y sus efectos, y arroyó resultados interesantes en términos de similitudes y variaciones en las formas y actuaciones del Estado. En cuanto a las similitudes, el resurgimiento de la escala nacional de regulación es un hecho prominente en ambos casos. Segundo, la forma de intervención es similar en términos de la imposición de las estrategias económicas neoliberales sobre los intereses del espacio vivido de los gobiernos locales. Tercero, ambos casos comparten el foco de intervención en términos de vivienda e infraestructura, vistas como mecanismo de estímulo a la economía y afianzamiento de la competitividad. Por último, se crearon nuevos y conflictivos espacios para el capital privado en el desarrollo urbano-regional.

Las implicaciones para la planeación urbana son más diversas. La recentralización del poder en el gobierno nacional implica el debilitamiento de los gobiernos locales, pero el desenvolvimiento concreto de este fenómeno muestra variantes importantes. En el caso del Reino Unido/Birmingham, se produjo una importante reestructuración legislativa e institucional del sistema mismo de planeación, incluyendo la creación de una nueva forma de la escala local o vecindario. En el caso de ColombiaMedellín, el gobierno central dejó esencialmente intacto el sistema de planeación, pero se impuso territorialmente a través de su poderío fiscal y nuevas agencias ejecutivas. En ambos casos el resultado ha sido el aumento en tensiones entre los niveles de gobierno y conflictos socio-territoriales.

Más allá de los efectos directos, la indagación de las transformaciones experimentadas en el campo de la planeación urbana pone en evidencia horizontes más amplios en cuanto al análisis del Estado. Las nuevas estrategias de intervención planificadora territarias 35 
después de la crisis financiera global dependían de la reconfiguración de las instituciones como dispositivos de poder regulatorio. No se trata de una simple reforma al sistema de planeación, sino más bien de una compleja reestructuración de las redes institucionales multiescalares mediante las cuales los intereses dominantes se concretizan en el espacio. Tal reestructuración involucra una amplia gama de instituciones y modos de regulación (jurídicos, financieros, comerciales, administrativos, policivos) que actúan colectivamente para asegurar nuevos espacios de rentabilidad y acumulación, y también para obstruir cualquier espacio para agendas urbanas que van en contra de este proceso. Comprender mejor esta situación conllevaría a una renovación del análisis crítico de la política urbana y de la ciudad como locus de poder, así como a una mejor fundamentación de la planeación progresista y de movimientos sociopolíticos desde abajo.

Agradecimiento: una primera versión de este trabajo fue presentada al congreso de la Asociación Europea de Escuelas de Planeación - AESOP (Dublin, 2013), con la coautoría de Paul Watson, Vice President Emeritus, Planning Officers Society (UK). Expreso mi reconocimiento y agradecimiento por su valiosa contribución.

\section{Referencias}

Aalbers, M. (2009). Geographies of the financial crisis. Area, 4l(1), 34-42.

Betancur, J. (2009). El segundo saqueo de América Latina: implicaciones para la planeación Urbana. En P. Brand (Ed.), La ciudad latinoamericana en el siglo XXI: globalización, neoliberalismo, planeación (pp. 83-120). Medellín: Universidad Nacional de Colombia.

Braithwaite, J. (2008). Regulatory Capitalism - How it Works, Ideas for Making it Work Better. Cheltenham: Edward Elgar.

Brenner, N. (2004). New State Spaces: urban governance and the re-scaling of statehood. Oxford: Oxford University Press.

Brenner, N. (2009). Open questions on state rescaling. Cambridge Journal of Regions, Economy and Society, 2,123-139.

Brenner, N., Peck J. \& Theodore N. (2010). Variegated neoliberalization: geographies, modalities, pathways. Global Networks, 10(2), 1-41.

Brenner, N. \& Theodore, N. (Eds.) (2002). Spaces of neoliberalism: urban restructuring in North America and Western Europe. Oxford: Blackwell.

Birmingham City Council (2012). Birmingham City Council's financial challenge, Birmingham City Council. Recuperado el 30 de marzo de 2013, de http://birminghamnewsroom. com/2012/10/birmingham-city-councils-financial-challenge/

Brand, P. \& Dávila, J. D. (2011). Mobility innovation at the urban margins. City, 15(6), 647-661. 
Brand, P. \& Prada, F. (2003). La invención de futuros urbanos. Medellin: Universidad Nacional de Colombia.

Conservative Party (2010). Open Source Planning, Policy Green Paper No. 14. London: Conservative Party.

Dávila, J. D. (Ed). (2013). Urban mobility and poverty. London: University College London, Universidad Nacional de Colombia.

Davoudi, S. \& Madanipour, A. (Eds.) (2015). Reconsidering Localism. London: Royal Town Planning Institute.

Department for Communities and Local Government (2010). Decentralisation and the Localism Bill: An essential guide, London: HMSO. Recuperado el 10 de abril de 2013, de https://www. gov.uk/government/publications / decentralisation-and-the-localism-billan-essential-guide--2

Eclac (2012a). The international financial crisis and its implications for Latin America and the Caribbean. Recuperado el 25 de abril de 2013, de http://www. eclac.org/

Eclac (2012b). Social panorama of Latin America 2012. Briefing Paper. Recuperado el 20 de abril de 2013, de http://www.cepal.org/cgi-bin/ getProd.asp? $\mathrm{xml}=/$ publicaciones / $\mathrm{xml} / 4 / 48454 / \mathrm{P} 48454 . \mathrm{xml} \& \mathrm{xsl}=/ \mathrm{tpl}-$ i/p9f.xsl\&base $=/$ tpl/top-bottom.xsl

Eclac (2013). Eclac predicts an upturn in economic growth for Latin America and the Caribbean in 2013. Recuperado el 25 de abril de 2013, de http://www. eclac.org
Harding, A., Harloe, M. \& Rees, J. (2010). Manchester's Bust Regime? International Journal of Urban and Regional Research, 34(4), 981-991.

Harvey, D. (2005). A brief history of neoliberalism. Oxford: Oxford University Press.

Hurtado, A., Torres, A. \& Mirando, L. (2011). El programa de sistemas integrados de transporte masivo en Colombia: ¿̨un ejemplo de recentralización de la gestión de las ciudades? Territorios, (25), 95-111.

Institute for Local Government Studies (2012). The world will be your oyster? Reflections on the Localism Act 2011. Birmingham: University of Birmingham.

Jessop, B. (2000). The crisis of the national spatio-temporal fix and the tendential ecological dominance of globalizing capitalism. International Journal of Urban and Regional Research, 24(2), 323-360.

Jessop, B. (1990). State theory: putting capitalist states in their place. Oxford: Blackwell.

Jessop, B., Brenner, N. \& Jones, M. (2008). Theorizing sociospatial relations. Environment and Planning D, 26, 389-401.

Jiménez, J.P. \& López, I. (2012). ¿¿Disminución de la desigualdad en América Latina? El rol de la política fiscal. Working Paper No. 33, Research Network on Interdependent Inequalities in America Latina. Recuperado el 25 de abril de 2013, de http://www.desigualdades. net/bilder/Working_Paper/33_WP_ Jimenez_Online.pdf territarias 35 
Joseph Rowntree Foundation (2012). Why are local authorities so nervous about their funding? Recuperado el 15 de marzo de 2013, de http:/ / www.jrf.org. uk/blog/2012/12/local-authoritiesfunding

Keil, R. (2010). Real estate, the city and place: the crisis unfolds. International Journal of Urban and Regional Research, 23(3), 647-651.

Kirkpatrick, O. \& Smith, M. (2011). The infrastructural limits to growth: rethinking the urban growth machine in times of fiscal crisis. International Journal of Urban and Regional Research, 35(3), 477-503.

Kitson, M., Martin, R. \& Tyler, P. (2011). The geographies of austerity. Cambridge Journal of Regions, Economy and Society, 4, 289-302.

Layard, A. (2012). The Localism Act 2011: What is 'local' and how do we (legally) construct it?. Environmental Law Review, 14(2): 134-144.

Levi-Faur, D. (2011). Regulation and regulatory governance. En D. Levi-Faur (Ed.), Handbook on the politics of regulation (pp. 3-21). Cheltenham: Edward Elgar.

Levitas, R. (2012). The Just's Umbrella: austerity and the Big Society in coalition policy and beyond. Critical Social Policy, 32(3), 320-342.

MacLeavy, J. (2011). “A 'new' politics of austerity, workfare and gender? The UK coalition government's welfare reform
Moncayo, V. (1990). Estado y espacialidad: formas y reformas. Bogotá: Universidad Nacional de Colombia.

Office for National Statistics (2014). Wealth in Great Britain Wave 3: 2020-2012 - Chapter 2 total wealth, may 2014. Recuperado el 10 de diciembre de 2014, de http://www.ons.gov.uk/ ons/rel/was/wealth-in-great-britainwave-3/2010-2012/report--chapter2--total-wealth.html

Peck, J., Theodore, N. \& Brenner, N. (2013). “Neoliberal Urbanism Redux?". International Journal of Urban and Regional Research, 37(3): 1091-9.

Peck, J. (2012). Austerity urbanism. City, $18(6), 626-655$.

Planning Officers Society (2012). Advice note on transition to the Localism Act and the National Planning Policy Framework. London: POS. Recuperado de: http:// www.planningofficers.org.uk/

Poulantzas, N. (1980). State, power, socialism. London: Verso

Pradilla, E. (1987) Capital, estado y vivienda en América Latina, México DF: Fontamara.

Raco, M. (2014). Delivering flagship projects in an era of regulatory capitalism: state-led privatization and the London Olympics 2012. International Journal of Urban and Regional Research, 38(1), 176-97.

Royal Town Planning Institute (2012). Map for England, London: RTPI Recuperado el 10 marzo 2013, de http://www. rtpi.org.uk/knowledge/core-issues/ map-for-england/ 
The Economist (2012). Gini back in the bottle: an unequal continent is becoming less so. Recuperado el 1 de junio de 2016 de: http://www.economist.com/node/21564411

The Guardian (2013a) Britain: a nation in decay. Recuperado el 8 de marzo 2013, de http://www.theguardian.com/commentisfree $/ 2013 / \mathrm{mar} / 08 /$ britaineconomy-long-term-fix

The Guardian (2013b). Council cuts will bring local government 'to its knees'. Recuperado el 26 de marzo de 2013 de http://www.guardian.co.uk/society $/ 2013 / \mathrm{mar} / 25 /$ council-cuts-localgovernment-knees

The Guardian $(2013 \mathrm{c})$. Cuts in council planning could let developers command agenda', Recuperado el 25 de marzo, de http://www.theguardian.com/ politics $/ 2013 / \mathrm{mar} / 25 /$ cuts-councilplanning-developers-command

Town and Country Planning Association (2013). The Lie of the Land!: England in the XXI century. Summary Report, London: TCPA. Recuperado el 1 de junio de 2016 de: http://www.tcpa.org. uk/data/files/Lie_of_the_Land_ExecSummary.pdf

Trade Union Council (2012). Perspectives on the Localism Act for union and community organisers and activists. Recuperado el 10 marzo 2013, de https://www.tuc. org.uk/tucfiles/354/Localism_Guide_2012.pdf

Vainer, C. (2011). Cidade de Exceção: reflexões a partir do Rio de Janeiro. XIV Encontro Nacional da Anpur. Rio de Janeiro. Recuperado el 10 marzo 2013, de https://br.boell.org/sites/default/ files/downloads/carlos_vainer_ippur_ cidade_de_excecao_reflexoes_a_partir_do_rio_de_janeiro.pdf

Wissen, M., \& Brand, U. (2011). Approaching the internationalization of the state: an Introduction. Antipode, 43(1), 1-11. 
